\title{
Prey Predator Fishery Model with Stage Structure in Two Patchy Marine Aquatic Environment
}

\author{
Mellachervu Naga Srinivas ${ }^{1}$, Mantripragada Ananata Satya Srinivas ${ }^{2}$, \\ Kalyan Das ${ }^{1}$, Nurul Huda Gazi ${ }^{3}$ \\ ${ }^{1}$ School of Advanced Sciences, Department of Mathematics, VIT University, Vellore, India \\ ${ }^{2}$ Department of Mathematics, Jawaharlal Nehru Technological University, Hyderabad, India \\ ${ }^{3}$ Department of Mathematics, Aliah University, Kolkata, India \\ E-mail: \{mnsrinivaselr,massrinivas\}@gmail.com, \{kalyandas70,nursha\}@rediffmail.com \\ Received July 4, 2011; revised August 2, 2011; accepted August 9, 2011
}

\begin{abstract}
In this paper, we propose and analyze a mathematical model to study the dynamics of a fishery resource system with stage structure in an aquatic environment that consists of two zones namely unreserved zone (fishing permitted) and reserved zone (fishing is strictly prohibited). In this model we introduce a stage structure in which predators are split into two kinds as immature predators and mature predators. It is assumed that immature predators cannot catch the prey and their foods are given by their parents (mature predators). It is also assumed that the fishing of immature predators prohibited in the unreserved zone and predator species are not allowed to enter inside the reserved zone. The local and global stability analysis has been specified. Biological and Bionomical equilibriums of the system are derived. Mathematical formulation of the optimal harvesting policy is given and its solution is derived in the equilibrium case by using Pontryagin's maximum principle.
\end{abstract}

Keywords: Prey Predator, Stage Structure, Local and Global Stability, Bionomic Equilibrium, Optimal Harvesting, Pontryagin's Maximum Principle

\section{Introduction}

There are numerous studies on the effects of harvesting on population growth. In the context of Predator-prey interaction, some studies that treat the populations being harvested as a homogeneous resource include those of Brauer and Soudack [1,2], Dai and Tang [3], Myerscough et al. [4], Chaudhuri [5] and Leung [6]. For a first look at the problem of harvesting from a bioeconomic or control theory point of view, see the works of Clark [7] and Levin et al. [8]. But they have not considered stage structure of species. Some of the stage structured models using time delay were considered by Aiello and Freedman [9], Freedman and Gopalsammy [10], Rosen [11], Fisher and Goh [12], Cushing and Saleem [13], and some other authors. In general, stage structured models exhibit much more complicated dynamics than ordinary models.

Bioeconomic modeling of the exploitation of biological resources such as fisheries and fore tries has gained importance in recent years. The techniques and issues associated with the bioeconomic exploitation of these resources have been discussed in detail by Clark [7,14]. Since most marine fisheries are essentially multi species in nature, exploitation of mixed species fisheries has started to draw attention from researchers. Although numerous models on single species fisheries have so far appeared in the fishery literature, no fully adequate studies on multispecies fisheries appear to exist.

It is very difficult to construct a realistic model of a multispecies community. Even if we succeed in formulating such a model, it is quite likely that the model may not be analytically tractable. Not every part of the catch is edible and harvesting harms some of the marine species which live on the other species in the sea. Thus the predator species are likely to become extinct with an indiscrete increase in the harvesting of prey species. Therefore, how best to harvest an ecologically or economically interdependent population in the sense of maximizing the present value of a stream of revenues from them, while maintaining ecological balance, is an important optimal control problem for fisheries.

Clark [14] discussed an optimal equilibrium policy for 
the combined harvesting of two ecologically independent species. Chaudhuri $[5,15]$ formulated an optimal control problem for the combined harvesting of two competing species. Models on the combined harvesting of a two species prey predator fishery have been discussed by Chaudhuri and Saha Ray [5], Mesterton-Gibbons [13], Ragozin and Brown [16] etc. Most of the mathematical models on the harvesting of a multispecies fishery have so far assumed that the species are affected by harvesting only

The prey-predator system is an important population model and has been studied by many authors [17-19]. It is assumed in the classical predator-prey model that each individual predator possesses the same ability to attack prey. Classical continuous population models such as logistic model and Volterra models overlook age structures and space structures. Also these models overlooked the rate at which mature predators attack the prey and the reproductive rate are also ignored. In [20] a stage structured model of one species growth consisting of immature and mature individuals was analyzed. In [21], it was further assumed that the time from immaturity to maturity is itself state dependent.

In recent years, the optimal management of renewable resources, which has a direct relationship to sustainable development, has been studied extensively by Clark [14], K. S. Chaudhuri [15], T. K. Kar, M. Swarnakamal [22] and W. Wang, L. Chen [23]. At present people are facing the problems due to shortage of resources. Extensive and unregulated harvesting of marine fishes can even lead to the extinction of several fish species. This problem can be addressed by arranging marine reserved zones, where fishing and other activities are prohibited. This marine reserve not only protects species inside the reserve area but also increase fish abundance in adjacent areas. The model of ecological system reflecting these problems has been given by T. K. Kar et al. [22] and Rui Zhang et al. [24].

Wendy-Wang et al. [25], considered prey-predator model with a stage structure in which predators are split in to immature predators and matured predators. They also assumed that the matured predators catch the prey and provide food for the immature predators. Rui Zhang et al. [24] considered a prey predator fishery model with prey dispersed in two patch environment, one is free zone for fishing and other is reserved zone where fishing is prohibited.

\section{The Mathematical Model}

Here we consider a habitat where prey and predator species are living together. It is assumed that the habitat is divided into two zones, namely, reserved and unreserved zones. It is also assumed that predator species are not allowed to enter inside the reserved zone whereas the free mixing of prey species from reserved to unreserved zone and vice-versa is permissible.

In the present paper we proposed a prey-predator model by combining the two features by $[24,25]$, in which prey dispersed in a two patch environment and predator is not allowed to enter inside the reserved zone. Also a stage-structure is incorporated in which predators are split in to immature and mature predators. Here it is assumed that the prey migrates from unreserved zone to reserved zone and vice-versa. It also assumed that the fishing of immature predators is prohibited in the unreserved zone. This paper deals with the following preypredator system

$$
\begin{aligned}
\frac{\mathrm{d} x_{1}}{\mathrm{~d} t}=r_{1} x_{1}- & \frac{r_{1} x_{1}^{2}}{k_{1}}-\beta x_{1} y_{2}-\sigma_{1} x_{1}+\sigma_{2} x_{2}-q_{1} E_{1} x_{1} \\
\frac{\mathrm{d} x_{2}}{\mathrm{~d} t}= & r_{2} x_{2}-\frac{r_{2} x_{2}^{2}}{k_{2}}+\sigma_{1} x_{1}-\sigma_{2} x_{2} \\
\frac{\mathrm{d} y_{1}}{\mathrm{~d} t} & =\frac{\alpha_{1} \beta x_{1} y_{2}^{2}}{w y_{1}+y_{2}}-\frac{\alpha_{2} \beta x_{1} y_{1} y_{2} w}{w y_{1}+y_{2}} \\
\frac{\mathrm{d} y_{2}}{\mathrm{~d} t} & =\frac{\alpha_{2} \beta x_{1} y_{1} y_{2} w}{w y_{1}+y_{2}}-d_{2} y_{2}-q_{2} E_{2} y_{2}
\end{aligned}
$$

Here $x_{1}(t), x_{2}(t)$ represents biomass densities of prey species in the unreserved and reserved areas respectively at a time " $t$ ". $y_{1}(t), y_{2}(t)$ represents biomass densities of immature and mature predators in unreserved area. $r_{1}, r_{2}$ represents intrinsic growth rates of prey species in unreserved and reserved areas respectively. $k_{1}, k_{2}$ represents carrying capacities of prey species in unreserved and reserved zones respectively. $\beta$ represents capturing rate or capturing efficiency of the predators. $\sigma_{1}, \sigma_{2}$ represents migration rates from unreserved to reserved zones and vice-versa. $q_{1}, q_{2}$ represents catch-ability coefficients of prey and matured predators respectively in unreserved zone. $E_{1}, E_{2}$ represents the efforts applied to harvest the prey and matured predator species respectively in unreserved zone. $d_{2}$ represents the death rate of mature predators in unreserved zone. $\alpha_{1}$ represents birth rate of predators. $\alpha_{2}$ represents conversion coefficient of immature predators to matured predators. We suppose that the attacking rate of mature predators at the prey i.e. the loss rate of prey is $\beta x_{1} y_{2}$.

Since mature predators and immature predators may have distinct consumption rates to the resource, we assume that they consume the resource in the ratio $w: 1$, where $w$ measures the relative consumption ratio between one immature predator and one mature predator, i.e., $w: 1$ gives the allocation ratio of food between one immature predator and one mature predator. We assume 
that all the weighted individuals share the quantity of food availability in equal parts. As a result, a fraction of resource consumed by mature predator is

$\beta x_{1} y_{2} \frac{y_{2}}{w y_{1}+y_{2}}$ and a fraction of resource consumed by immature predators is $\beta x_{1} y_{2} \frac{w y_{1}}{w y_{1}+y_{2}}$.

If there is no migration of fish population from the reserved area to the unreserved area (i.e. $\left.\sigma_{2}=0\right)$ and

$r_{1}-\sigma_{1}-q_{1} E_{1}<0$, then $\frac{\mathrm{d} x_{1}}{\mathrm{~d} t}<0$. Similarly if there is no migration of fish population from the unreserved area to reserved area (i.e. $\sigma_{1}=0$ ) and $r_{2}-\sigma_{2}<0$, then $\frac{\mathrm{d} x_{2}}{\mathrm{~d} t}<0$. Therefore we assume that $r_{1}-\sigma_{1}-q_{1} E_{1}>0$, $r_{2}-\sigma_{2}>0$ throughout our analysis.

\section{Existence of Equilibria}

The steady state equations of (2.1)-(2.4) are

$$
\begin{gathered}
r_{1} x_{1}-\frac{r_{1} x_{1}^{2}}{k_{1}}-\beta x_{1} y_{2}-\sigma_{1} x_{1}+\sigma_{2} x_{2}-q_{1} E_{1} x_{1}=0 \\
r_{2} x_{2}-\frac{r_{2} x_{2}^{2}}{k_{2}}+\sigma_{1} x_{1}-\sigma_{2} x_{2}=0 \\
\alpha_{1} \beta x_{1} y_{2} \frac{y_{2}}{w y_{1}+y_{2}}-\alpha_{2} \beta x_{1} y_{2} \frac{w y_{1}}{w y_{1}+y_{2}}=0 \\
\alpha_{2} \beta x_{1} y_{2} \frac{w y_{1}}{w y_{1}+y_{2}}-d_{2} y_{2}-q_{2} E_{2} y_{2}=0
\end{gathered}
$$

The three possible equilibrium points are

1) $G_{1}\left(x_{1}^{*}, x_{2}^{*}, 0,0\right)$ (In the absence of predators in unreserved zone);

2) $G_{2}\left(x_{1}^{\phi}, x_{2}^{\phi}, y_{1}^{\phi}, 0\right)$ (In the absence of mature predators in unreserved zone);

3) $G_{3}\left(\bar{x}_{1}, \bar{x}_{2}, \bar{y}_{1}, \bar{y}_{2}\right)$ (The interior equilibrium).

Case 1): $G_{1}\left(x_{1}^{*}, x_{2}^{*}, 0,0\right)$ :

In this case, $x_{1}^{*}, x_{2}^{*}$ are the positive solutions of (3.1) $\&$ (3.2). It may be noted that for $x_{1}^{*}, x_{2}^{*}$ to be positive we must have

$$
\begin{gathered}
\frac{r_{2}\left(r_{1}-\sigma_{1}-q_{1} E_{1}\right)}{k_{2} \sigma_{2}^{2}}<\frac{\left(r_{2}-\sigma_{2}\right) r_{1}}{k_{1} \sigma_{2}} \\
\frac{\left(r_{2}-\sigma_{2}\right)\left(r_{1}-\sigma_{1}-q_{1} E_{1}\right)}{\sigma_{2}}<\sigma_{1} \\
\frac{r_{1} x_{1}^{*}}{k_{1}}>\left(r_{1}-\sigma_{1}-q_{1} E_{1}\right) .
\end{gathered}
$$

Case 2): In the absence of mature predators in the un- reserved zone, there will not be any production of immature predators and hence this case coincides with Case 1).

Case 3): Solving (3.1), (3.2), (3.3), \& (3.4), we get

$$
\begin{gathered}
\bar{x}_{1}=\frac{1}{\alpha_{1} \alpha_{2} \beta}\left(\alpha_{1}+\alpha_{2}\right)\left(d_{2}+q_{2} E_{2}\right) \\
\bar{x}_{2}=\frac{k_{2}}{2 r_{2}}\left[\left(r_{2}-\sigma_{2}\right)+\sqrt{\left(r_{2}-\sigma_{2}\right)^{2}+\frac{4 r_{2} \sigma_{1} \bar{x}_{1}}{k_{2}}}\right] \\
\bar{y}_{1}=\frac{\alpha_{1}}{\alpha_{2} \beta \bar{x}_{1} w}\left[\left(r_{1}-\sigma_{1}-q_{1} E_{1}\right) \bar{x}_{1}-\frac{r_{1} \bar{x}_{1}^{2}}{k_{1}}+\sigma_{2} \bar{x}_{2}\right] \\
\bar{y}_{2}=\frac{1}{\beta \bar{x}_{1}}\left[\left(r_{1}-\sigma_{1}-q_{1} E_{1}\right) \bar{x}_{1}-\frac{r_{1} \bar{x}_{1}^{2}}{k_{1}}+\sigma_{2} \bar{x}_{2}\right]
\end{gathered}
$$

For $\bar{y}_{1}$ and $\bar{y}_{2}$ both to be positive, we must have

$$
\left(r_{1}-\sigma_{1}-q_{1} E_{1}\right) \bar{x}_{1}+\sigma_{2} \bar{x}_{2}>\frac{r_{1} \bar{x}_{1}^{2}}{k_{1}} .
$$

\section{Qualitative Analysis of the Model}

\subsection{Local Stability Analysis}

Let us now suppose that system (2.1)-(2.4) has a unique equilibrium $G_{3}\left(\bar{x}_{1}, \bar{x}_{2}, \bar{y}_{1}, \bar{y}_{2}\right)$.

$$
J=\left[\begin{array}{llll}
J_{11} & J_{12} & J_{13} & J_{14} \\
J_{21} & J_{22} & J_{23} & J_{24} \\
J_{31} & J_{32} & J_{33} & J_{34} \\
J_{41} & J_{42} & J_{43} & J_{44}
\end{array}\right]
$$

where

$$
\begin{aligned}
& J_{11}=r_{1}-\frac{2 r_{1} \bar{x}_{1}}{k_{1}}-\beta \bar{y}_{2}-\sigma_{1}-q_{1} E_{1}=\frac{-r_{1} \bar{x}_{1}}{k_{1}}-\frac{\sigma_{2} \bar{x}_{2}}{\bar{x}_{1}} \\
& J_{21}=\sigma_{1} ; \\
& J_{31}=\frac{\alpha_{1} \beta \bar{y}_{2}^{2}}{w \bar{y}_{1}+\bar{y}_{2}}-\frac{\alpha_{2} \beta \bar{y}_{1} \bar{y}_{2} w}{w \bar{y}_{1}+\bar{y}_{2}} ; \\
& J_{41}=\frac{\alpha_{2} \beta \bar{y}_{1} \bar{y}_{2} w}{w \bar{y}_{1}+\bar{y}_{2}} ; \\
& J_{12}=\sigma_{2} ; \\
& J_{22}=r_{2}-\frac{2 r_{2} \bar{x}_{2}}{k_{2}}-\sigma_{2}=\frac{-r_{2} \bar{x}_{2}}{k_{2}}-\frac{\sigma_{1} \bar{x}_{1}}{\bar{x}_{2}} ; J_{32}=0 ; \\
& J_{42}=0 ; \quad J_{13}=0 ; \quad J_{23}=0 ; \\
& J_{33}=-\frac{\alpha_{1} \beta \bar{x}_{1} \bar{y}_{2}^{2} w}{\left(w \bar{y}_{1}+\bar{y}_{2}\right)^{2}}-\frac{\alpha_{2} \beta \bar{x}_{1}\left(\bar{y}_{2}\right)^{2} w}{\left(w \bar{y}_{1}+\bar{y}_{2}\right)^{2}} ;
\end{aligned}
$$




$$
\begin{aligned}
& J_{43}=\frac{\alpha_{2} \beta \bar{x}_{1}\left(\bar{y}_{2}\right)^{2} w}{\left(w \bar{y}_{1}+\bar{y}_{2}\right)^{2}} ; \\
& J_{14}=-\beta \bar{x}_{1} ; \\
& J_{24}=0 ; \\
& J_{34}=\frac{2 \alpha_{1} \beta \bar{x}_{1} \bar{y}_{1} \bar{y}_{2} w}{\left(w \bar{y}_{1}+\bar{y}_{2}\right)^{2}}+\frac{\alpha_{1} \beta \bar{x}_{1}\left(\bar{y}_{2}\right)^{2}}{\left(w \bar{y}_{1}+\bar{y}_{2}\right)^{2}}-\frac{\alpha_{2} \beta \bar{x}_{1}\left(\bar{y}_{1}\right)^{2} w^{2}}{\left(w \bar{y}_{1}+\bar{y}_{2}\right)^{2}} ; \\
& J_{44}=\frac{\alpha_{2} \beta \bar{x}_{1}\left(\bar{y}_{1}\right)^{2} w^{2}}{\left(w \bar{y}_{1}+\bar{y}_{2}\right)^{2}}-d_{2}-q_{2} E_{2}=-\frac{\alpha_{2} \beta \bar{x}_{1} w \bar{y}_{1} \bar{y}_{2}}{\left(w \bar{y}_{1}+\bar{y}_{2}\right)^{2}} .
\end{aligned}
$$

The characteristic equation of the Jacobian matrix of (2.1)-(2.4) at $G_{3}\left(\bar{x}_{1}, \bar{x}_{2}, \bar{y}_{1}, \bar{y}_{2}\right)$ is

$$
\lambda^{4}+a_{1} \lambda^{3}+a_{2} \lambda^{2}+a_{3} \lambda+a_{4}=0
$$

where

$$
\begin{gathered}
a_{1}=\left(\frac{r_{1} x_{1}}{k_{1}}+\frac{r_{2} x_{2}}{k_{2}}+\frac{\sigma_{2} x_{2}}{x_{1}}+\frac{\sigma_{1} x_{1}}{x_{2}}\right) \\
+\frac{\beta x_{1} y_{2} w}{p^{2}}\left(\alpha_{1} y_{2}+\alpha_{2} y_{1}+\alpha_{2} y_{2}\right)>0 \\
a_{2}=\left(\frac{r_{1} r_{2} x_{1} x_{2}}{k_{1} k_{2}}+\frac{r_{1} \sigma_{1} x_{1}{ }^{2}}{k_{1} x_{2}}+\frac{r_{2} \sigma_{2} x_{2}{ }^{2}}{k_{2} x_{1}}\right) \\
+\frac{\alpha_{2} \beta^{2} x_{1}{ }^{2} y_{1} y_{2}{ }^{3} w^{2}}{p^{4}}\left[2\left(\alpha_{1}+\alpha_{2}\right)\right]+\frac{\alpha_{2} \beta^{2} x_{1} y_{1} y_{2} w}{p} \\
+\left[\frac{r_{2} \beta x_{1} x_{2} y_{2} w}{k_{2} p^{2}}+\frac{\sigma_{1} \beta x_{1}{ }^{2} y_{2} w}{x_{2} p^{2}}+\frac{r_{1} \beta x_{1}{ }^{2} y_{2} w}{k_{1} p^{2}}+\frac{\sigma_{2} \beta x_{1} y_{2} w}{p^{2}}\right] \\
\cdot\left[\alpha_{1} y_{2}+\alpha_{2} y_{2}+\alpha_{2} y_{1}\right]>0 \\
a_{3}=\left(\frac{r_{1} r_{2} x_{1} x_{2}}{k_{1} k_{2}}+\frac{r_{1} \sigma_{1} x_{1}{ }^{2}}{k_{1} x_{2}}+\frac{r_{2} \sigma_{2} x_{2}{ }^{2}}{x_{1} k_{2}}\right) \\
\cdot\left[\left(\alpha_{1}+\alpha_{2}\right) \frac{\beta x_{1} y_{2}{ }^{2} w}{p^{2}}+\frac{\alpha_{2} \beta x_{1} y_{1} y_{2} w}{p^{2}}\right] \\
+\frac{\alpha_{2} \beta^{2} x_{1} y_{1} y_{2} w}{p}\left[\left(\alpha_{1}+\alpha_{2}\right) \frac{\beta x_{1} y_{2}{ }^{2} w}{p^{2}}+\left(\frac{r_{2} x_{2}}{k_{2}}+\frac{\sigma_{1} x_{1}}{x_{2}}\right)\right]>0 \\
a_{4}=\frac{\alpha_{2} \beta^{3} x_{1}{ }^{2} y_{1} y_{2}{ }^{3} w^{2}}{p^{3}}\left(\alpha_{1}+\alpha_{2}\right)\left(\frac{r_{2} x_{2}}{k_{2}}+\frac{\sigma_{1} x_{1}}{x_{2}}\right)>0 .
\end{gathered}
$$

Now,

$$
\begin{aligned}
& a_{1}=\left(\frac{r_{1} x_{1}}{k_{1}}+\frac{r_{2} x_{2}}{k_{2}}+\frac{\sigma_{2} x_{2}}{x_{1}}+\frac{\sigma_{1} x_{1}}{x_{2}}\right) \\
& +\frac{\beta x_{1} y_{2} w}{p^{2}}\left(\alpha_{1} y_{2}+\alpha_{2} y_{1}+\alpha_{2} y_{2}\right) ; \\
& =A+\left(\frac{\beta x_{1} y_{2} w}{p^{2}}\right) B
\end{aligned}
$$

where

$$
\begin{gathered}
A=\left(\frac{r_{1} x_{1}}{k_{1}}+\frac{r_{2} x_{2}}{k_{2}}+\frac{\sigma_{2} x_{2}}{x_{1}}+\frac{\sigma_{1} x_{1}}{x_{2}}\right) ; \\
B=\alpha_{1} y_{2}+\alpha_{2} y_{1}+\alpha_{2} y_{2} \\
a_{2}=\frac{r_{1} r_{2} x_{1} x_{2}}{K_{1} K_{2}}+\frac{r_{1} \sigma_{1} x_{1}^{2}}{K_{1} x_{2}}+\frac{r_{2} \sigma_{2} x_{2}^{2}}{K_{2} x_{1}} \\
a_{2}=\frac{r_{1} r_{2} x_{1} x_{2}}{K_{1} K_{2}}+\frac{r_{1} \sigma_{1} x_{1}^{2}}{K_{1} x_{2}}+\frac{r_{2} \sigma_{2} x_{2}^{2}}{K_{2} x_{1}} \\
+\left[\frac{r_{2} \beta x_{1} x_{2} y_{2} w}{K_{2} p^{2}}+\frac{\sigma_{1} \beta x_{1} y_{2} w}{x_{2} p^{2}}+\frac{r_{1} \beta x_{1}{ }^{2} y_{2} w}{K_{1} p^{2}}+\frac{\sigma_{2} \beta x_{2} y_{2} w}{p^{2}}\right] \\
+\left(\alpha_{1} y_{2}+\alpha_{2} y_{2}+\alpha_{2} y_{1}\right) \\
+\frac{\alpha_{2} \beta^{2} x_{1}{ }^{2} y_{1} y_{2}{ }^{3} w^{2}}{p^{4}}\left[2\left(\alpha_{1}+\alpha_{2}\right)\right]+\frac{\alpha_{2} \beta^{2} x_{1} y_{1} y_{2} w}{p} \\
=C+\left(\frac{\beta x_{1} y_{2} w}{p^{2}}\right) A\left(\alpha_{1} y_{2}+\alpha_{2} y_{2}+\alpha_{2} y_{1}\right) \\
+\frac{\alpha_{2} \beta^{2} x_{1}{ }^{2} y_{1} y_{2}{ }^{3} w^{2}}{p^{4}} 2\left(\alpha_{1}+\alpha_{2}\right)+\frac{\alpha_{2} \beta^{2} x_{1} y_{1} y_{2} w}{p}
\end{gathered}
$$

where

$$
\begin{gathered}
C=\frac{r_{1} r_{2} x_{1} x_{2}}{k_{1} k_{2}}+\frac{r_{1} \sigma_{1} x_{1}^{2}}{k_{1} x_{2}}+\frac{r_{2} \sigma_{2} x_{2}^{2}}{k_{2} x_{1}} \\
a_{3}=\left(\frac{r_{1} r_{2} x_{1} x_{2}}{k_{1} k_{2}}+\frac{r_{1} \sigma_{1} x_{1}^{2}}{k_{1} x_{2}}+\frac{r_{2} \sigma_{2} x_{2}^{2}}{x_{1} k_{2}}\right) \\
\quad \cdot\left[\left(\alpha_{1}+\alpha_{2}\right) \frac{\beta x_{1} y_{2}{ }^{2} w}{p^{2}}+\frac{\alpha_{2} \beta x_{1} y_{1} y_{2} w}{p^{2}}\right] \\
+\frac{\alpha_{2} \beta^{2} x_{1} y_{1} y_{2} w}{p}\left[\left(\alpha_{1}+\alpha_{2}\right) \frac{\beta x_{1} y_{2}{ }^{2} w}{p^{2}}+\left(\frac{r_{2} x_{2}}{k_{2}}+\frac{\sigma_{1} x_{1}}{x_{2}}\right)\right] \\
a_{4}=\frac{\alpha_{2} \beta^{3} x_{1}^{2} y_{1} y_{2}^{3} w^{2}}{p^{3}}\left(\alpha_{1}+\alpha_{2}\right)\left(\frac{r_{2} x_{2}}{k_{2}}+\frac{\sigma_{1} x_{1}}{x_{2}}\right)>0 .
\end{gathered}
$$

Again,

$$
\begin{aligned}
& \left(a_{1} a_{2}-a_{3}\right)=A \cdot C+A^{2}\left(\frac{\beta x_{1} y_{2} w}{p^{2}}\right) B \\
& +A \frac{\alpha_{2} \beta^{2} x_{1}{ }^{2} y_{1} y_{2}{ }^{3} w^{2}}{p^{4}} 2\left(\alpha_{1}+\alpha_{2}\right) \\
& +\frac{\alpha_{2} \beta^{2} x_{1} y_{1} y_{2} w}{p}\left(\frac{r_{1} x_{1}}{k_{1}}+\frac{\sigma_{2} x_{2}}{x_{1}}\right) \\
& +C\left(\frac{\beta x_{1} y_{2} w}{p^{2}}\right) B+\frac{\beta^{2} x_{1}{ }^{2} y_{2}{ }^{2} w^{2}}{p^{4}} A B^{2} \\
& +\frac{\alpha_{2} \beta^{3} x_{1}^{3} y_{1} y_{2}{ }^{4} w^{3}}{p^{6}} 2\left(\alpha_{1}+\alpha_{2}\right) B+\frac{\alpha_{2}{ }^{2} \beta^{3} x_{1}{ }^{2} y_{1}^{2} y_{2}{ }^{2} w^{2}}{p^{3}} .
\end{aligned}
$$

Therefore, 


$$
\begin{aligned}
& \left(a_{1} a_{2}-a_{3}\right) a_{3}=\left[A^{2}\left(\frac{r_{2} x_{2}}{k_{2}}+\frac{\sigma_{1} x_{1}}{x_{2}}\right) \frac{\alpha_{2} \beta^{3} x_{1}^{2} y_{1} y_{2}^{2} w^{2}}{p^{3}} B\right] \\
& +\left[A \cdot \frac{\alpha_{2} \beta^{5} x_{1}^{4} y_{1} y_{2}^{5} w^{4}}{p^{7}}\left(\alpha_{1}+\alpha_{2}\right) B^{2}\right] \\
& +\left[A \cdot \frac{\alpha_{2} \beta^{4} x_{1}^{3} y_{1} y_{2}{ }^{3} w^{3}}{p^{5}}\left(\frac{r_{2} x_{2}}{k_{2}}+\frac{\sigma_{1} x_{1}}{x_{2}}\right) B^{2}\right] \\
& +\left[A^{2} \cdot \frac{\alpha_{2} \beta^{4} x_{1} y_{1} y_{2}{ }^{4} w^{3}}{p^{5}}\left(\alpha_{1}+\alpha_{2}\right) B\right]+\left[A \cdot C^{2}\left(\frac{\beta x_{1} y_{2} w}{p^{2}}\right) \cdot B\right] \\
& +\left[A \cdot C \cdot \frac{\alpha_{2} \beta^{3} x_{1}{ }^{2} y_{1} y_{2}{ }^{3} w^{2}}{p^{3}}\left(\alpha_{1}+\alpha_{2}\right)\right] \\
& +\left[A \cdot C \cdot\left(\frac{r_{2} x_{2}}{k_{2}}+\frac{\sigma_{1} x_{1}}{x_{2}}\right)\left(\frac{\alpha_{2} \beta^{2} x_{1} y_{1} y_{2} w}{p}\right)\right] \\
& +\left[A^{2} \cdot C \cdot\left(\frac{\beta^{2} x_{1}^{2} y_{2}^{2} w^{2}}{p^{4}}\right) B^{2}\right] \\
& +\left[A \cdot C \cdot \frac{\alpha_{2} \beta^{3} x_{1}^{3} y_{1} y_{2}{ }^{4} w^{3}}{p^{6}} 2\left(\alpha_{1}+\alpha_{2}\right) B\right] \\
& +\left[A \cdot \frac{\alpha_{2}^{2} \beta^{5} x_{1}^{4} y_{1}^{2} y_{2}{ }^{6} w^{4}}{p^{7}} 2\left(\alpha_{1}+\alpha_{2}\right)^{2}\right] \\
& +\left[A \cdot \frac{\alpha_{2}{ }^{2} \beta^{4} x_{1}^{3} y_{1}^{2} y_{2}{ }^{4} w^{3}}{p^{5}} 2\left(\alpha_{1}+\alpha_{2}\right)\left(\frac{r_{2} x_{2}}{k_{2}}+\frac{\sigma_{1} x_{1}}{x_{2}}\right)\right] \\
& +\left[C \frac{\alpha_{2} \beta^{3} x_{1}^{2} y_{1} y_{2}^{2} w^{2}}{p^{3}}\left(\frac{r_{1} x_{1}}{k_{1}}+\frac{\sigma_{2} x_{2}}{x_{1}}\right) B\right] \\
& +\left[\frac{\alpha_{2}^{2} \beta^{4} x_{1}^{3} y_{1}{ }^{2} y_{2}{ }^{4} w^{3}}{p^{4}}\left(\alpha_{1}+\alpha_{2}\right)\left(\frac{r_{1} x_{1}}{k_{1}}+\frac{\sigma_{2} x_{2}}{x_{1}}\right)\right] \\
& +\left[\frac{\alpha_{2}^{2} \beta^{4} x_{1}^{2} y_{1}^{2} y_{2}^{2} w^{2}}{p^{2}}\left(\frac{r_{1} x_{1}}{k_{1}}+\frac{\sigma_{2} x_{2}}{x_{1}}\right)\left(\frac{r_{2} x_{2}}{k_{2}}+\frac{\sigma_{1} x_{1}}{x_{2}}\right)\right] \\
& +\left[A \cdot C \cdot \frac{\beta^{3} x_{1}^{3} y_{2}{ }^{3} w^{3}}{p^{6}} B^{3}\right] \\
& +\left[C \cdot \frac{\alpha_{2} \beta^{4} x_{1}{ }^{4} y_{1} y_{2}{ }^{5} w^{4}}{p^{8}} 2\left(\alpha_{1}+\alpha_{2}\right) B^{2}\right] \\
& +\left[\frac{\alpha_{2}^{2} \beta^{6} x_{1}^{5} y_{1}^{2} y_{2}^{7} w^{5}}{p^{9}} 2\left(\alpha_{1}+\alpha_{2}\right)^{2} B\right] \\
& +\left[\frac{\alpha_{2}^{2} \beta^{5} x_{1}^{4} y_{1}^{2} y_{2}^{5} w^{4}}{p^{7}} 2\left(\alpha_{1}+\alpha_{2}\right) B\left(\frac{r_{2} x_{2}}{k_{2}}+\frac{\sigma_{1} x_{1}}{x_{2}}\right)\right] \\
& +\left[C \cdot \frac{\alpha_{2}^{2} \beta^{4} x_{1}^{3} y_{1}^{2} y_{2}^{3} w^{3}}{p^{5}} B\right]+\left[\frac{\alpha_{2}^{3} \beta^{6} x_{1}^{4} y_{1}^{3} y_{2}^{5} w^{4}}{p^{5}}\left(\alpha_{1}+\alpha_{2}\right)\right] \\
& +\left[\frac{\alpha_{2}^{3} \beta^{5} x_{1}^{3} y_{1}^{3} y_{2}^{3} w^{3}}{p^{4}}\left(\frac{r_{2} x_{2}}{k_{2}}+\frac{\sigma_{1} x_{1}}{x_{2}}\right)\right] \text {. }
\end{aligned}
$$

Again,

$$
\begin{aligned}
a_{1}{ }^{2} a_{4}= & {\left[A^{2}+\frac{\beta^{2} x_{1}{ }^{2} y_{2}{ }^{2} w^{2}}{p^{4}} B^{2}+2 A \cdot \frac{\beta x_{1} y_{2} w}{p^{2}} B\right] } \\
& \cdot\left(\frac{r_{2} x_{2}}{k_{2}}+\frac{\sigma_{1} x_{1}}{x_{2}}\right)\left(\frac{\alpha_{2} \beta^{3} x_{1}{ }^{2} y_{1} y_{2}{ }^{3} w^{2}}{p^{3}}\right)\left(\alpha_{1}+\alpha_{2}\right) \\
\Rightarrow a_{1}{ }^{2} a_{4}= & A^{2}\left(\frac{r_{2} x_{2}}{k_{2}}+\frac{\sigma_{1} x_{1}}{x_{2}}\right)\left(\alpha_{1}+\alpha_{2}\right) \\
& \cdot\left(\frac{\alpha_{2} \beta^{3} x_{1}{ }^{2} y_{1} y_{2}{ }^{3} w^{2}}{p^{3}}\right) B^{2} \cdot\left(\frac{\alpha_{2} \beta^{5} x_{1}{ }^{4} y_{1} y_{2}{ }^{5} w^{4}}{p^{7}}\right) \\
& \cdot\left(\frac{r_{2} x_{2}}{k_{2}}+\frac{\sigma_{1} x_{1}}{x_{2}}\right)\left(\alpha_{1}+\alpha_{2}\right) 2 A \cdot \frac{\alpha_{2} \beta^{4} x_{1}^{3} y_{1} y_{2}{ }^{4} w^{3}}{p^{5}} \\
& \cdot B\left(\alpha_{1}+\alpha_{2}\right)\left(\frac{r_{2} x_{2}}{k_{2}}+\frac{\sigma_{1} x_{1}}{x_{2}}\right) .
\end{aligned}
$$

By combining first four terms of the right hand side of (4.3) with the first three terms of right hand side of (4.4), it can easily be established that

$$
\left(a_{1} a_{2}-a_{3}\right) a_{3}-a_{1}^{2} a_{4}>0 .
$$

Since $a_{4}>0$, it is clear that

$$
a_{4}\left(a_{1} a_{2} a_{3}-a_{1}^{2} a_{4}-a_{3}^{2}\right)>0 .
$$

Hence $G_{4}\left(\bar{x}_{1}, \bar{x}_{2}, \bar{y}_{1}, \bar{y}_{2}\right)$ is locally asymptotically stable. So by Routh-Hurwitz criteria, it follows that all eigen values of (3.2) have negative real parts if andonly if $a_{1}>0, a_{3}>0, a_{4}>0, \quad a_{3}\left(a_{1} a_{2}-a_{3}\right)>a_{1}^{2} a_{4}$ and $a_{4}\left(a_{1} a_{2} a_{3}-a_{1}^{2} a_{4}-a_{3}^{2}\right)>0$.

Hence, $G_{3}\left(\bar{x}_{1}, \bar{x}_{2}, \bar{y}_{1}, \bar{y}_{2}\right)$ is Locally asymptotically stable. Thus the four populations remain stable under the conditions we have obtained in the above study. The fact can also be proved numerically. We have solved the above system for a set of parameter values. The parameter values also satisfy the condition for existence of the interior equilibrium point $G_{3}\left(\bar{x}_{1}, \bar{x}_{2}, \bar{y}_{1}, \bar{y}_{2}\right)$. The real data is very difficult to obtain. So we use a set of the hypothetical parameter values as follows: $r_{1}=1.6 ; r_{2}=$ $1.2 ; k_{1}=270 ; k_{2}=250 ; \sigma_{1}=0.4 ; \sigma_{2}=0.4 ; q_{1}=3.0 ; q_{2}=$ $3.2 ; E_{1}=0.02 ; E_{2}=0.082 ; \alpha_{1}=1.9 ; \alpha_{2}=2.1 ; \beta=0.06 ; w$ $=0.7 ; d_{2}=0.22$. The time series of the populations are shown in the Figure 1. The Figure 1 shows that the populations are finite for long time and the system is stable.

\subsection{Global Stability Analysis}

Theorem I. The Equilibrium point $G_{1}\left(x_{1}^{*}, x_{2}^{*}, 0,0\right)$ is globally asymptotically stable.

Proof: let us consider the following Lyapunov function 

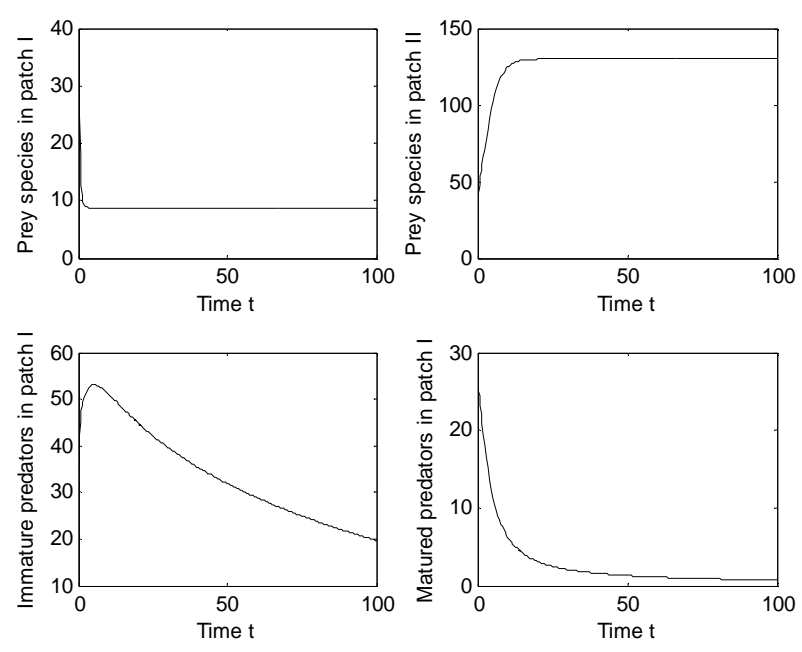

Figure 1. Stable time series evolution of the prey and predator populations of the model system (2.1)-(2.4).

$$
\begin{aligned}
& V\left(x_{1}, x_{2}\right)=\left(x_{1}-x_{1}^{*}-x_{1}^{*} \ln \left(\frac{x_{1}}{x_{1}^{*}}\right)\right)+l_{1}\left(x_{2}-x_{2}^{*}-x_{2}^{*} \ln \left(\frac{x_{2}}{x_{2}^{*}}\right)\right) \\
& V\left(x_{1}, x_{2}, y_{1}, y_{2}\right)=\left(x_{1}-\bar{x}_{1}-\bar{x}_{1} \ln \left(\frac{x_{1}}{\bar{x}_{1}}\right)\right)+l_{1}\left(x_{2}-\bar{x}_{2}-\bar{x}_{2} \ln \left(\frac{x_{2}}{\bar{x}_{2}}\right)\right)+l_{2}\left(y_{1}-\bar{y}_{1}-\bar{y}_{1} \ln \left(\frac{y_{1}}{\bar{y}_{1}}\right)\right)+l_{3}\left(y_{2}-\bar{y}_{2}-\bar{y}_{2} \ln \left(\frac{y_{2}}{\bar{y}_{2}}\right)\right) \\
& \Rightarrow \frac{\mathrm{d} V}{\mathrm{~d} t}=\left(\frac{x_{1}-\bar{x}_{1}}{x_{1}}\right) \frac{\mathrm{d} x_{1}}{\mathrm{~d} t}+l_{1}\left(\frac{x_{2}-\bar{x}_{2}}{x_{2}}\right) \frac{\mathrm{d} x_{2}}{\mathrm{~d} t}+l_{2}\left(\frac{y_{1}-\bar{y}_{1}}{y_{1}}\right) \frac{\mathrm{d} y_{1}}{\mathrm{~d} t}+l_{3}\left(\frac{y_{2}-\bar{y}_{2}}{y_{2}}\right) \frac{\mathrm{d} y_{2}}{\mathrm{~d} t} \\
& \Rightarrow \frac{\mathrm{d} V}{\mathrm{~d} t}=\left(x_{1}-\bar{x}_{1}\right)\left[r_{1}-\frac{r_{1} x_{1}}{k_{1}}-\beta y_{2}-\sigma_{1}+\frac{\sigma_{2} x_{2}}{x_{1}}-q_{1} E_{1}\right]+l_{1}\left(x_{2}-\bar{x}_{2}\right)\left[r_{2}-\frac{r_{2} x_{2}}{k_{2}}+\frac{\sigma_{1} x_{1}}{x_{2}}-\sigma_{2}\right] \\
& +l_{2}\left(y_{1}-\bar{y}_{1}\right)\left[\alpha_{1} \beta x_{1} y_{2} \frac{y_{2}}{y_{1}\left(w y_{1}+y_{2}\right)}-\alpha_{2} \beta x_{1} w \frac{y_{2}}{\left(w y_{1}+y_{2}\right)}\right]+l_{3}\left(y_{2}-\bar{y}_{2}\right)\left[\alpha_{2} \beta x_{1} \frac{w y_{1}}{\left(w y_{1}+y_{2}\right)}-d_{2}-q_{2} E_{2}\right] \\
& \Rightarrow \frac{\mathrm{d} V}{\mathrm{~d} t}=\left(x_{1}-\bar{x}_{1}\right)\left[-\frac{r_{1}}{k_{1}}\left(x_{1}-\bar{x}_{1}\right)+\sigma_{2}\left(\frac{x_{2}}{x_{1}}-\frac{\bar{x}_{2}}{\bar{x}_{1}}\right)-\beta\left(y_{2}-\bar{y}_{2}\right)\right]+l_{1}\left(x_{2}-\bar{x}_{2}\right)\left[-\frac{r_{2}}{k_{2}}\left(x_{2}-\bar{x}_{2}\right)+\sigma_{1}\left(\frac{x_{1}}{x_{2}}-\frac{\bar{x}_{1}}{\bar{x}_{2}}\right)\right] \\
& +l_{2}\left(y_{1}-\bar{y}_{1}\right) \frac{\alpha_{1} \beta x_{1} y_{2}}{\left(w y_{1}+y_{2}\right)}\left(\frac{y_{2}}{y_{1}}-\frac{\bar{y}_{2}}{\bar{y}_{1}}\right)+l_{3}\left(y_{2}-\bar{y}_{2}\right) \alpha 2 \beta w\left[\frac{x_{1} y_{1}}{\left(w y_{1}+y_{2}\right)}-\frac{\bar{x}_{1} \bar{y}_{1}}{\left(w \bar{y}_{1}+\bar{y}_{2}\right)}\right] \text {. }
\end{aligned}
$$

Choosing $l_{1}=\frac{\bar{x}_{2} \sigma_{2}}{\bar{x}_{1} \sigma_{1}} ; \quad l_{2}=\frac{1}{\alpha_{1}} ; l_{3}=\frac{w \bar{y}_{1}+\bar{y}_{2}}{\alpha_{2} w \bar{y}_{1}}$

$$
\begin{aligned}
\Rightarrow \frac{\mathrm{d} V}{\mathrm{~d} t}= & -\frac{r_{1}}{k_{1}}\left(x_{1}-\bar{x}_{1}\right)^{2}+\frac{\sigma_{2}\left(x_{1}-\bar{x}_{1}\right)}{x_{1} \bar{x}}\left(x_{2} \bar{x}_{1}-x_{1} \bar{x}_{2}\right)-\beta\left(x_{1}-\bar{x}_{1}\right)\left(y_{2}-\bar{y}_{2}\right)-\frac{r_{2} \bar{x}_{2} \sigma_{2}}{k_{2} \bar{x}_{1} \sigma_{1}}\left(x_{2}-\bar{x}_{2}\right)^{2} \\
& +\frac{\bar{x}_{2} \sigma_{2}}{\bar{x}_{1} \sigma_{1}} \sigma_{1} \frac{\left(x_{2}-\bar{x}_{2}\right)}{x_{2} \bar{x}_{2}}\left(x_{1} \bar{x}_{2}-x_{2} \bar{x}_{1}\right)+\frac{1}{\alpha_{1}}\left[\frac{\alpha_{1} \beta x_{1} y_{2}}{y_{1} \bar{y}_{1}\left(w y_{1}+y_{2}\right)}\left(y_{1}-\bar{y}_{1}\right)\left(y_{2} \bar{y}_{1}-y_{1} \bar{y}_{2}\right)\right] \\
& +\frac{w \bar{y}_{1}+\bar{y}_{2}}{\alpha_{2} w \bar{y}_{1}}\left[\alpha 2 \beta w\left(y_{2}-\bar{y}_{2}\right)\left(\frac{x_{1} y_{1}}{\left(w y_{1}+y_{2}\right)}-\frac{\bar{x}_{1} \bar{y}_{1}}{\left(w \bar{y}_{1}+\bar{y}_{2}\right)}+\frac{x_{1} \bar{y}_{1}}{\left(w \bar{y}_{1}+\bar{y}_{2}\right)}-\frac{x_{1} \bar{y}_{1}}{\left(w \bar{y}_{1}+\bar{y}_{2}\right)}\right)\right] \\
\Rightarrow \frac{\mathrm{d} V}{\mathrm{~d} t}= & -\frac{r_{1}}{k_{1}}\left(x_{1}-\bar{x}_{1}\right)^{2}-\frac{r_{2} \bar{x}_{2} \sigma_{2}}{k_{2} \bar{x}_{1} \sigma_{1}}\left(x_{2}-\bar{x}_{2}\right)^{2}-\frac{\sigma_{2}}{x_{1} x_{2} \bar{x}_{1}}\left(x_{2} \bar{x}_{1}-x_{1} \bar{x}_{2}\right)^{2}-\frac{\beta x_{1}}{y_{1} \bar{y}_{1}\left(w y_{1}+y_{2}\right)}\left(y_{2} \bar{y}_{1}-y_{1} \bar{y}_{2}\right)^{2}<0 .
\end{aligned}
$$


Therefore $G_{3}\left(\bar{x}_{1}, \bar{x}_{2}, \bar{y}_{1}, \bar{y}_{2}\right)$ is globally asymptotically stable.

\section{Qualitative Bionomic Phenomena}

It is the study of the dynamics of living resources using economic models. Let $c_{1}$ be the fishing cost per unit effort for prey species, $c_{2}$ be the fishing cost per unit effort for matured predator species, $p_{1}$ be the price per unit biomass of the prey, $p_{2}$ be the price per unit biomass of the predator (matured). Therefore net revenue or economic rent at any time given by $R=R_{1}+R_{2}$ where $R_{1}=\left(p_{1} q_{1} x_{1}-c_{1}\right) E_{1}, R_{2}=\left(p_{2} q_{2} y_{2}-c_{2}\right) E_{2}$; here $R_{1}$ represents Net Revenue for the prey; $R_{2}$ represents Net revenue for matured predator species.

The bionomic equilibrium

$\left(\left(x_{1}\right)_{\infty},\left(x_{2}\right)_{\infty},\left(y_{1}\right)_{\infty},\left(y_{2}\right)_{\infty},\left(E_{1}\right)_{\infty},\left(E_{2}\right)_{\infty}\right)$ is given by the following equations

$$
\begin{gathered}
r_{1} x_{1}-\frac{r_{1} x_{1}^{2}}{k_{1}}-\beta x_{1} y_{2}-\sigma_{1} x_{1}+\sigma_{2} x_{2}-q_{1} E_{1} x_{1}=0 \\
r_{2} x_{2}-\frac{r_{2} x_{2}^{2}}{k_{2}}+\sigma_{1} x_{1}-\sigma_{2} x_{2}=0 \\
\frac{\alpha_{1} \beta x_{1} y_{2}^{2}}{w y_{1}+y_{2}}-\frac{\alpha_{2} \beta x_{1} y_{1} y_{2} w}{w y_{1}+y_{2}}=0 \\
\frac{\alpha_{2} \beta x_{1} y_{1} y_{2} w}{w y_{1}+y_{2}}-d_{2} y_{2}-q_{2} E_{2} y_{2}=0 \\
R=\left(p_{1} q_{1} x_{1}-c_{1}\right) E_{1}+\left(p_{2} q_{2} y_{2}-c_{2}\right) E_{2}=0
\end{gathered}
$$

In order to determine the bionomic equilibrium we come across the following cases.

Case I. If for the matured predator, fishing cost is greater than the revenue $\left(c_{2}>p_{2} q_{2} y_{2}\right)$, then fishing of matured predator is not feasible. Hence fishing of prey population remains operational $\left(c_{1}<p_{1} q_{1} x_{1}\right)$.

Thus, when $E_{2}=0$ and $c_{1}<p_{1} q_{1} x_{1}$ we have

$$
\begin{gathered}
\left(x_{1}\right)_{\infty}=\frac{c_{1}}{p_{1} q_{1}}, \\
\left(x_{2}\right)_{\infty}=\frac{k_{2}}{2 r_{2}}\left[\left(r_{2}-\sigma_{2}\right)+\sqrt{\left(r_{2}-\sigma_{2}\right)+\frac{4 r_{2} \sigma_{1} c_{1}}{p_{1} q_{1} k_{2}}}\right] .
\end{gathered}
$$

Since $c_{1}<p_{1} q_{1} x_{1}<p_{1} q_{1} k_{1}, \quad\left(\left(y_{2}\right)_{\infty},\left(E_{1}\right)_{\infty}\right)$ will be any point on the line

$$
\begin{aligned}
& \sigma_{1}+\beta y_{2}+q_{1} E_{1}=r_{1}\left(1-\frac{c_{1}}{p_{1} q_{1} k_{1}}\right) \\
& +\frac{\sigma_{2} p_{1} q_{1} k_{2}}{2 r_{2} c_{1}}\left[\left(r_{2}-\sigma_{2}\right)+\sqrt{\left(r_{2}-\sigma_{2}\right)+\frac{4 r_{2} \sigma_{1} c_{1}}{p_{1} q_{1} k_{2}}}\right] .
\end{aligned}
$$

Case II. If $c_{1}>p_{1} q_{1} x_{1}$ i.e., the cost is greater than the revenue in the prey fishing, then the prey fishing will be closed (i.e. $E_{1}=0$ ). Only matured predator fishing remains operational (i.e. $\left.c_{2}<p_{2} q_{2} y_{2}\right)$

$$
\left(y_{2}\right)_{\infty}=\frac{c_{2}}{p_{2} q_{2}} .
$$

Substitute this value in (5.1), we get

$$
\left(x_{2}\right)_{\infty}=\frac{1}{\sigma_{2}}\left[\frac{\beta c_{2}\left(x_{1}\right)_{\infty}}{p_{2} q_{2}}+\sigma_{1}\left(x_{1}\right)_{\infty}-r_{1}\left(x_{1}\right)_{\infty}\left(1-\frac{\left(x_{1}\right)_{\infty}}{k_{1}}\right)\right] \text {. }
$$

Here $\left(x_{1}\right)_{\infty}$ is the positive solution of

where

$$
a_{1} x_{1}^{3}+a_{2} x_{1}^{2}+a_{3} x_{1}+a_{4}=0
$$

$$
\begin{aligned}
& a_{1}=\frac{r_{1}^{2} r_{2}}{k_{1}^{2} k_{2} \sigma_{2}^{2}}>0 ; \\
& a_{2}=-\frac{2 r_{1} r_{2}}{k_{1} k_{2} \sigma_{2}^{2}}\left[r_{1}-\sigma_{1}-\frac{\beta c_{2}}{p_{2} q_{2}}\right] \text {; } \\
& a_{3}=\frac{r_{2}}{k_{2} \sigma_{2}^{2}}\left(r_{1}-\sigma_{1}-\frac{\beta c_{2}}{p_{2} q_{2}}\right)^{2}-\frac{r_{1}}{k_{1} \sigma_{2}}\left(r_{2}-\sigma_{2}\right) \text {; } \\
& a_{4}=\frac{1}{\sigma_{2}}\left(r_{2}-\sigma_{2}\right)\left(r_{1}-\sigma_{1}-\frac{\beta c_{2}}{p_{2} q_{2}}\right)-\sigma_{1} \text {. } \\
& \text { Now if }\left[r_{1}-\sigma_{1}-\frac{\beta c_{2}}{p_{2} q_{2}}\right]<0 \text { (or) }\left[r_{1}-\sigma_{1}-\frac{\beta c_{2}}{p_{2} q_{2}}\right]>0 \text {, } \\
& \text { then } \frac{r_{2}}{k_{2} \sigma_{2}^{2}}\left(r_{1}-\sigma_{1}-\frac{\beta c_{2}}{p_{2} q_{2}}\right)^{2}<\frac{r_{1}\left(r_{2}-\sigma_{2}\right)}{k_{1} \sigma_{2}} \\
& \text { and }\left(r_{2}-\sigma_{2}\right)\left(r_{1}-\sigma_{1}-\frac{\beta c_{2}}{p_{2} q_{2}}\right)<\sigma_{1} \sigma_{2} \text {. }
\end{aligned}
$$

Then Equation (5.6) has a unique positive solution $x_{1}=\left(x_{1}\right)_{\infty}$

For $\left(x_{2}\right)_{\infty}$ to be positive, we must have

$$
\left(x_{1}\right)_{\infty}>k_{1}-\frac{\beta c_{2} k_{1}}{p_{2} q_{2} r_{1}}-\frac{\sigma_{1} k_{1}}{r_{1}} .
$$

Substitute the value of $\left(x_{1}\right)_{\infty}$ in Equation (5.4), we get

$$
\left(E_{2}\right)_{\infty}=\frac{1}{q_{2}}\left[\frac{\alpha_{2} \beta\left(x_{1}\right)_{\infty}\left(y_{1}\right)_{\infty} w}{w\left(y_{1}\right)_{\infty}+\left(y_{2}\right)_{\infty}}-d_{2}\right]
$$

$\left(E_{2}\right)_{\infty}>0$, Provided $\frac{\alpha_{1} \alpha_{2} \beta\left(x_{1}\right)_{\infty}}{\alpha_{1}+\alpha_{2}}>d_{2}$

where $w=\frac{\alpha_{1}\left(y_{2}\right)_{\infty}}{\alpha_{2}\left(y_{1}\right)_{\infty}}$. 
Case III. If $c_{1}>p_{1} q_{1} x_{1}, c_{2}>p_{2} q_{2} y_{2}$, then the cost is greater than revenues for both the species and the whole fishery will be closed.

Case IV. If $c_{1}<p_{1} q_{1} x_{1}, c_{2}<p_{2} q_{2} y_{2}$, then the revenues for both the species being positive, then the whole fishery will be in operation.

In this case

$$
\begin{gathered}
\left(x_{1}\right)_{\infty}=\frac{c_{1}}{p_{1} q_{1}} \\
\left(y_{2}\right)_{\infty}=\frac{c_{2}}{p_{2} q_{2}} .
\end{gathered}
$$

Substitute (4.7) and (4.8) in (4.1), (4.2), (4.4) we get

$$
\begin{aligned}
&\left(x_{2}\right)_{\infty}=\frac{k_{2}}{2 r_{2}}\left[\left(r_{2}-\sigma_{2}\right)+\sqrt{\left(r_{2}-\sigma_{2}\right)+\frac{4 r_{2} \sigma_{1} c_{1}}{p_{1} q_{1} k_{2}}}\right]\left(E_{1}\right)_{\infty}=\frac{r_{1}}{q_{1}}\left[1-\frac{c_{1}}{p_{1} q_{1} k_{1}}\right]-\frac{\beta c_{2}}{q_{1} p_{2} q_{2}}-\frac{\sigma_{1}}{q_{1}}+\frac{\sigma_{2}\left(x_{2}\right)_{\infty} p_{1}}{q_{1} c_{1}} \\
&\left(E_{2}\right)_{\infty}=\frac{1}{q_{2}}\left[\frac{\alpha_{2} \beta\left(x_{1}\right)_{\infty} y_{1} w}{w y_{1}+y_{2}}-d_{2}\right] \\
&\left(E_{1}\right)_{\infty}>0 \text { if } \\
& \frac{r_{1}}{q_{1}}\left[1-\frac{c_{1}}{p_{1} q_{1} k_{1}}\right]+\frac{\sigma_{2}\left(x_{2}\right)_{\infty} p_{1}}{q_{1} c_{1}}>\frac{\beta c_{2}}{q_{1} p_{2} q_{2}}+\frac{\sigma_{1}}{q_{1}} \\
&\left(E_{2}\right)_{\infty}>0 \text { if } \frac{\alpha_{1} \alpha_{2}}{\alpha_{1}+\alpha_{2}} \frac{\beta c_{1}}{p_{1} q_{1}}>d_{2} .
\end{aligned}
$$

The Non-trivial Bionomic equilibrium point $\left(\left(x_{1}\right)_{\infty},\left(x_{2}\right)_{\infty},\left(y_{2}\right)_{\infty},\left(E_{1}\right)_{\infty},\left(E_{2}\right)_{\infty}\right)$ exists if (5.12) and (5.13) hold.

\section{Optimal Harvesting Policy}

In this section we study optimal harvesting policy of the system (2.1)-(2.4). Also in this section we employ the Pontryagin's maximum principle to obtain a path of optimal harvesting policy so that if the fish populations inside and outside the reserve zones, are kept along this path, then the regulatory agency is assured to achieve its objective. We consider the following present value $J$ of a continuous time-stream

$$
J=\int_{0}^{\infty} P\left(x_{1}, x_{2}, y_{1}, y_{2}, E_{1}, E_{2}, t\right) \mathrm{e}^{-\delta t} \mathrm{~d} t
$$

where $P$ is the net revenue given by

$$
\begin{aligned}
& P\left(x_{1}, x_{2}, y_{1}, y_{2}, E_{1}, E_{2}, t\right) \\
& =\left(p_{1} q_{1} x_{1} E_{1}-c_{1} E_{1}\right)+\left(p_{2} q_{2} y_{2} E_{2}-c_{2} E_{2}\right)
\end{aligned}
$$

and $\delta$ denotes the instantaneous annual rate of dis- count, the aim of this section is to maximize $J$ subjected to the state Equations (2.1)-(2.4).

Firstly we construct the following Hamiltonian function

$$
\begin{aligned}
& H=\mathrm{e}^{-\delta t}\left[p_{1} q_{1} x_{1}-c_{1}\right] E_{1}+\mathrm{e}^{-\delta t}\left[p_{2} q_{2} y_{2}-c_{2}\right] E_{2} \\
& +\lambda_{1}\left[r_{1} x_{1}\left(1-\frac{x_{1}}{k_{1}}\right)-\beta x_{1} y_{2}-\sigma_{1} x_{1}+\sigma_{2} x_{2}-q_{1} E_{1} x_{1}\right] \\
& +\lambda_{2}\left[r_{2} x_{2}\left(1-\frac{x_{2}}{k_{2}}\right)+\sigma_{1} x_{1}-\sigma_{2} x_{2}\right] \\
& +\lambda_{3}\left[\alpha_{1} \beta x_{1} y_{2} \frac{y_{2}}{w y_{1}+y_{2}}-\alpha_{2} \beta x_{1} y_{2} \frac{w y_{1}}{w y_{1}+y_{2}}\right] \\
& +\lambda_{4}\left[\alpha_{2} \beta x_{1} y_{2} \frac{w y_{1}}{w y_{1}+y_{2}}-d_{2} y_{2}-q_{2} E_{2} y_{2}\right]
\end{aligned}
$$

where $\lambda_{1}, \lambda_{2}, \lambda_{3}, \lambda_{4}$ are additional unknown functions called the adjoint variables, $E_{1}, E_{2}$ are the control variables satisfying the constraints $0 \leq E_{1} \leq\left(E_{1}\right)_{\text {max }}$;

$0 \leq E_{2} \leq\left(E_{2}\right)_{\max }$, and

$\phi_{1}(t)=\mathrm{e}^{-\delta t}\left(p_{1} q_{1} x_{1}-c_{1}\right)-\lambda_{1} q_{1} x_{1}$,

$\phi_{2}(t)=\mathrm{e}^{-\delta t}\left(p_{2} q_{2} y_{2}-c_{2}\right)-\lambda_{4} q_{2} y_{2}$ are called the switching functions.

We aim to find an optimal equilibrium

$\left(\left(x_{1}\right)_{\delta},\left(x_{2}\right)_{\delta},\left(y_{1}\right)_{\delta},\left(y_{2}\right)_{\delta},\left(E_{1}\right)_{\delta},\left(E_{2}\right)_{\delta}\right)$ to maximize Hamiltonian $H$.

Since Hamiltonian $H$ is linear in the control variables $E_{1}, E_{2}$, the optimal control can be extreme controls or the singular controls, thus we have

$E_{1}=\left(E_{1}\right)_{\max }$, when $\phi_{1}(t)>0$

i.e., when $\lambda_{1}(t) \mathrm{e}^{\delta t}<p_{1}-\frac{c_{1}}{q_{1} x_{1}}$;

$E_{1}=0$, when $\phi_{1}(t)<0$

i.e., when $\lambda_{1}(t) \mathrm{e}^{\delta t}>p_{1}-\frac{c_{1}}{q_{1} x_{1}}$;

$E_{2}=\left(E_{2}\right)_{\max }$, when $\phi_{2}(t)>0$

i.e., when $\lambda_{4}(t) \mathrm{e}^{\delta t}<p_{2}-\frac{c_{2}}{q_{2} y_{2}}$;

$E_{2}=0$, when $\phi_{2}(t)<0$

i.e., when $\lambda_{4}(t) \mathrm{e}^{\delta t}>p_{2}-\frac{c_{2}}{q_{2} y_{2}}$;

when $\phi_{1}(t)=0, \lambda_{1}(t) \mathrm{e}^{\delta t}=p_{1}-\frac{c_{1}}{q_{1} x_{1}} ;$

or

$$
\frac{\partial H}{\partial E_{1}}=0
$$


when $\phi_{2}(t)=0, \lambda_{4}(t) \mathrm{e}^{\delta t}=p_{2}-\frac{c_{2}}{q_{2} y_{2}} ;$

or

$$
\frac{\partial H}{\partial E_{2}}=0
$$

In this case, the optimal control is called the singular control, and (6.4), (6.5) are the necessary conditions for the maximization of Hamiltonian $H$.

By Pontrayagin's maximal principle, the adjoint equations are

$$
\begin{aligned}
& \frac{\mathrm{d} \lambda_{1}}{\mathrm{~d} t}=-\frac{\partial H}{\partial x_{1}} \\
& =-\left[\mathrm{e}^{-\delta \mathrm{t}} \mathrm{p}_{1} \mathrm{q}_{1} \mathrm{E}_{1}+\lambda_{1}\left(\mathrm{r}_{1}-\frac{2 \mathrm{r}_{1} \mathrm{x}_{1}}{k_{1}}-\beta y_{2}-\sigma_{1}-q_{1} E_{1}\right)\right. \\
& \left.+\lambda_{2} \sigma_{1}+\lambda_{3}\left(\frac{\alpha_{1} \beta\left(y_{2}\right)^{2}}{w y_{1}+y_{2}}-\frac{\alpha_{2} \beta w y_{1} y_{2}}{w y_{1}+y_{2}}\right)+\lambda_{4}\left(\frac{\alpha_{2} \beta w y_{1} y_{2}}{w y_{1}+y_{2}}\right)\right] \\
& \frac{\mathrm{d} \lambda_{2}}{\mathrm{~d} t}=-\frac{\partial H}{\partial x_{2}}=-\left[\lambda_{1} \sigma_{2}+\lambda_{2}\left(r_{2}-\frac{2 r_{2} x_{2}}{k_{2}}-\sigma_{2}\right)\right] \\
& \frac{\mathrm{d} \lambda_{3}}{\mathrm{~d} t}=-\frac{\partial H}{\partial y_{1}} \\
& =-\left[\lambda_{3}\left(-\frac{\alpha_{1} \beta x_{1} y_{2}^{2} w}{\left(w y_{1}+y_{2}\right)^{2}}-\frac{\alpha_{2} \beta x_{1} y_{2} w y_{2}}{\left(w y_{1}+y_{2}\right)^{2}}\right)\right. \\
& \left.+\lambda_{4}\left(\frac{\alpha_{2} \beta x_{1} y_{2} w y_{2}}{\left(w y_{1}+y_{2}\right)^{2}}\right)\right] \\
& \frac{\mathrm{d} \lambda_{4}}{\mathrm{~d} t}=-\frac{\partial H}{\partial y_{2}} \\
& =-\left[\mathrm{e}^{-\delta t} p_{2} q_{2} E_{2}+\lambda_{1}\left(-\beta x_{1}\right)\right. \\
& +\lambda_{3}\left(\frac{\alpha_{1} \beta x_{1}\left(2 y_{1} y_{2} w+y_{2}^{2}\right)}{\left(w y_{1}+y_{2}\right)^{2}}-\frac{\alpha_{2} \beta x_{1} w^{2} y_{1}^{2}}{\left(w y_{1}+y_{2}\right)^{2}}\right) \\
& \left.+\lambda_{4}\left(\frac{\alpha_{2} \beta x_{1} w^{2} y_{1}^{2}}{\left(w y_{1}+y_{2}\right)^{2}}-d_{2}-q_{2} E_{2}\right)\right] \text {. }
\end{aligned}
$$

Considering the interior equilibrium $G_{3}\left(\bar{x}_{1}, \bar{x}_{2}, \bar{y}_{1}, \bar{y}_{2}\right)$ and Equations (6.4), (6.7), can be written as

$\frac{\mathrm{d} \lambda_{2}}{\mathrm{~d} t}-\lambda_{2}\left(A_{1}\right)=-A_{2} \mathrm{e}^{-\delta t}$

where $A_{1}=\frac{r_{2} \bar{x}_{2}}{k_{2}}+\frac{\sigma_{1} \bar{x}_{1}}{\bar{x}_{2}} ; \quad A_{2}=\left(p_{1}-\frac{c_{1}}{q_{1} \bar{x}_{1}}\right) \sigma_{2}$.

We can calculate that

$$
\lambda_{2}=\frac{A_{2}}{A_{1}+\delta} \mathrm{e}^{-\delta t}
$$

Similarly, by considering the interior equilibrium $G_{3}\left(\bar{x}_{1}, \bar{x}_{2}, \bar{y}_{1}, \bar{y}_{2}\right)$ and Equations (6.5), (6.8), can be written as $\frac{\mathrm{d} \lambda_{3}}{\mathrm{~d} t}-\lambda_{3} A_{3}=-\mathrm{e}^{-\delta t} A_{4}$

where

$$
\begin{aligned}
& A_{3}=\frac{\alpha_{2} \beta w \bar{x}_{1} \bar{y}_{2}}{w \bar{y}_{1}+\bar{y}_{2}} ; \\
& A_{4}=\left(p_{2}-\frac{c_{2}}{q_{2} \bar{y}_{2}}\right)\left(\frac{\alpha_{2} \beta w \bar{x}_{1} \bar{y}_{2}^{2}}{\left(w \bar{y}_{1}+\bar{y}_{2}\right)^{2}}\right) .
\end{aligned}
$$

We can conclude that

$$
\lambda_{3}=\frac{A_{4}}{A_{3}+\delta} \mathrm{e}^{-\delta t} .
$$

Similarly, by considering the interior equilibrium $G_{3}\left(\bar{x}_{1}, \bar{x}_{2}, \bar{y}_{1}, \bar{y}_{2}\right)$ and Equations (6.10), (6.11), (6.6) can be written as $\frac{\mathrm{d} \lambda_{1}}{\mathrm{~d} t}-\lambda_{1} A_{5}=-\mathrm{e}^{-\delta t} A_{6}$

where

$$
\begin{aligned}
A_{5}= & \frac{r_{1} \bar{x}_{1}}{k_{1}}+\frac{\sigma_{2} \bar{x}_{2}}{\bar{x}_{1}} \\
A_{6}= & p_{1} q_{1} E_{1}+\frac{A_{2}}{A_{1}+\delta} \sigma_{1}+\frac{A_{4}}{A_{3}+\delta}\left(\alpha_{1} \bar{y}_{2}-\alpha_{2} w \bar{y}_{1}\right) \\
& +\left(p_{2}-\frac{c_{2}}{q_{2} \bar{y}_{2}}\right)\left(\frac{\alpha_{2} \beta w \bar{y}_{1} \bar{y}_{2}}{w \bar{y}_{1}+\bar{y}_{2}}\right) .
\end{aligned}
$$

We can conclude that

$$
\lambda_{1}=\frac{A_{6}}{A_{5}+\delta} \mathrm{e}^{-\delta t} .
$$

Similarly by considering the interior equilibrium $G_{3}\left(\bar{x}_{1}, \bar{x}_{2}, \bar{y}_{1}, \bar{y}_{2}\right)$ and Equations (6.10), (6.11), (6.6) can be written as $\frac{\mathrm{d} \lambda_{4}}{\mathrm{~d} t}-\lambda_{4} A_{7}=-\mathrm{e}^{-\delta t} A_{8}$ where

$$
\begin{aligned}
& A_{7}=\frac{\alpha_{2} \beta \bar{x}_{1} w^{2} \bar{y}_{1}^{2}}{\left(w \bar{y}_{1}+\bar{y}_{2}\right)^{2}}-d_{2}-q_{2} E_{2} \\
& A_{8}=p_{2} q_{2} E_{2}-\frac{A_{6}}{A_{5}+\delta} \beta \bar{x}_{1}+\frac{A_{4}}{A_{3}+\delta}\left(\frac{\alpha_{2} \beta \bar{x}_{1} w \bar{y}_{2}}{w \bar{y}_{1}+\bar{y}_{2}}\right) .
\end{aligned}
$$

We can calculate that

$$
\lambda_{4}=\frac{A_{8}}{A_{7}+\delta} \mathrm{e}^{-\delta t} .
$$

It is obviously that $\lambda_{1}(t), \lambda_{2}(t), \lambda_{3}(t), \lambda_{4}(t)$ are bounded as $t \rightarrow \infty$.

Substitute (6.12) in (6.4), we obtain a singular path

$$
p_{1}-\frac{c_{1}}{q_{1} \bar{x}_{1}}=\frac{A_{6}}{A_{5}+\delta} .
$$

Substitute (6.13) in (6.5), we obtain a singular path 


$$
p_{2}-\frac{c_{2}}{q_{2} \bar{y}_{2}}=\frac{A_{8}}{A_{7}+\delta} .
$$

Using

$$
\begin{aligned}
& \bar{x}_{2}=\frac{k_{2}}{2 r_{2}}\left[\left(r_{2}-\sigma_{2}\right)+\sqrt{\left(r_{2}-\sigma_{2}\right)^{2}+\frac{4 r_{2} \sigma_{1} \bar{x}_{1}}{k_{2}}}\right] \\
& \bar{y}_{2}=\frac{1}{\beta \bar{x}_{1}}\left[\left(r_{1}-\sigma_{1}-q_{1} E_{1}\right) \bar{x}_{1}-\frac{r_{1} \bar{x}_{1}^{2}}{k_{1}}+\sigma_{2} \bar{x}_{2}\right],
\end{aligned}
$$

$A_{1}, A_{2}, A_{3}, A_{4}, A_{5}, A_{6}, A_{7}, A_{8}$ can be written as

$$
\begin{aligned}
& A_{1}=\frac{1}{2}\left(r_{2}-\sigma_{2}\right)+\frac{1}{2}\left[\left(r_{2}-\sigma_{2}\right)^{2}+\frac{4 r_{2} \sigma_{1} \bar{x}_{1}}{k_{2}}\right]^{\frac{1}{2}} \\
& +\frac{2 r_{2} \sigma_{1} \bar{x}_{1}}{k_{2}\left[\left(r_{2}-\sigma_{2}\right)+\sqrt{\left(r_{2}-\sigma_{2}\right)^{2}+\frac{4 r_{2} \sigma_{1} \bar{x}_{1}}{k_{2}}}\right]^{1 / 2}} \\
& A_{2}=\left(p_{1}-\frac{c_{1}}{q_{1} \bar{x}_{1}}\right) \sigma_{2} \\
& A_{3}=\frac{\alpha_{1} \alpha_{2} \beta w \bar{x}_{1}}{\alpha_{1}+\alpha_{2}}\left(p_{1}-\frac{c_{1}}{q_{1} \bar{x}_{1}}\right) \sigma_{2} \\
& A_{4}=p_{2}-\frac{c_{2}}{\frac{q_{2}}{\beta \bar{x}_{1}}\left[\left(r_{1}-\sigma_{1}-q_{1} E_{1}\right) \bar{x}_{1}-\frac{r_{1} \bar{x}_{1}^{2}}{k_{1}}+\sigma_{2} \bar{x}_{2}\right]} \\
& A_{5}=\frac{r_{1} \bar{x}_{1}}{k_{1}}+\frac{\sigma_{2} k_{2}}{2 \bar{x}_{1} r_{2}}\left[\left(r_{2}-\sigma_{2}\right)+\left(\left(r_{2}-\sigma_{2}\right)^{2}+\frac{4 r_{2} \sigma_{1} \bar{x}_{1}}{k_{2}}\right)^{1 / 2}\right] \\
& A_{6}=p_{1} q_{1} E_{1}+\frac{\left(p_{1}-\frac{c_{1}}{q_{1} \bar{x}_{1}}\right) \sigma_{2}}{\left(\frac{r_{2} \bar{x}_{2}}{k_{2}}+\frac{\sigma_{1} \bar{x}_{1}}{2 \bar{x}_{2}}\right)+\delta} \\
& +\left(p_{2}-\frac{c_{2} \beta \bar{x}_{1}}{q_{2}\left[\left(r_{2}-\sigma_{2}-q_{1} E_{1}\right) \bar{x}_{1}-\frac{r_{1} \bar{x}_{1}^{2}}{k_{1}}+\sigma_{2} \bar{x}_{2}\right]}\right)\left[\frac{\alpha_{2}^{2} \beta w \bar{y}_{1}}{\left(\alpha_{1}+\alpha_{2}\right)}\right] \\
& A_{7}=\frac{\alpha_{1}^{2} \alpha_{2} \beta \bar{x}_{1}}{\left(\alpha_{1}+\alpha_{2}\right)^{2}}-d_{2}-q_{2} E_{2} \\
& A_{8}=p_{2} q_{2} E_{2}-\frac{A_{6}}{A_{5}+\delta} \beta \bar{x}_{1}+\frac{A_{4}}{A_{3}+\delta}\left(\frac{\alpha_{2}^{2} \beta \bar{x}_{1} w}{\alpha_{1}+\alpha_{2}}\right) \text {. }
\end{aligned}
$$

Thus (6.14) and (6.15) can be written as

$$
F\left(\bar{x}_{1}\right)=\left(p_{1}-\frac{c_{1}}{q_{1} \bar{x}_{1}}\right)-\frac{A_{6}}{A_{5}+\delta}
$$

and $G\left(\bar{y}_{2}\right)=\left(p_{2}-\frac{c_{2}}{q_{2} \bar{y}_{2}}\right)-\frac{A_{8}}{A_{7}+\delta}$.
There exists a unique positive root $\bar{x}_{1}=\left(x_{1}\right)_{\delta}$ of $F\left(\bar{x}_{1}\right)=0$ in the interval $0<\bar{x}_{1}<k_{1}$ If the following hold $F(0)<0, F\left(k_{1}\right)>0, F^{\prime}\left(\bar{x}_{1}\right)>0$ for $\bar{x}_{1}>0$, and Similarly There exists a unique positive root $\bar{y}_{2}=\left(y_{2}\right)_{\delta}$ of $G\left(\bar{y}_{2}\right)=0$ in the interval $0<\bar{y}_{2}<k_{2}$ If the following hold $G(0)<0, G\left(k_{2}\right)>0, G^{\prime}\left(\bar{y}_{2}\right)>0$ for $\bar{y}_{2}>0$.

For $\bar{x}_{1}=\left(x_{1}\right)_{\delta}, \bar{y}_{2}=\left(y_{2}\right)_{\delta}$,

we get

$$
\begin{aligned}
& \left(x_{2}\right)_{\delta}=\frac{k_{2}}{2 r}\left[\left(r_{2}-\sigma_{2}\right)+\sqrt{\left(r_{2}-\sigma_{2}\right)^{2}+\frac{4 r_{2} \sigma_{1} \bar{x}_{1}}{k_{2}}}\right] \\
& \left(y_{2}\right)_{\delta}=\frac{\alpha_{2} w\left(y_{1}\right)_{\delta}}{\alpha_{1}} ; \\
& \left(y_{1}\right)_{\delta}=\frac{\alpha_{1}}{\alpha_{2} \beta \bar{x}_{1} w}\left[\left(r_{1}-\sigma_{1}-q_{1} E_{1}\right) \bar{x}_{1}-\frac{r_{1}\left(\bar{x}_{1}\right)_{\delta}^{2}}{k_{1}}+\sigma_{2}\left(\bar{x}_{2}\right)_{\delta}\right] \\
& \left(E_{1}\right)_{\delta}=\frac{1}{q_{1}\left(x_{1}\right)_{\delta}} \\
& \cdot\left[r_{1}\left(x_{1}\right)_{\delta}\left(1-\frac{\left(x_{1}\right)_{\delta}}{k_{1}}\right)-\beta\left(x_{1}\right)_{\delta}\left(y_{2}\right)_{\delta}-\sigma_{1}\left(x_{1}\right)_{\delta}-\sigma_{2}\left(x_{2}\right)_{\delta}\right] \\
& \left(E_{2}\right)_{\delta}=\frac{1}{q_{2}\left(y_{2}\right)_{\delta}}\left[\frac{\alpha_{2} \beta\left(x_{1}\right)_{\delta}\left(y_{1}\right)_{\delta}\left(y_{2}\right)_{\delta} w}{w\left(y_{1}\right)_{\delta}+\left(y_{2}\right)_{\delta}}-d_{2}\left(y_{2}\right)_{\delta}\right] \\
& \text { i.e., }\left(E_{2}\right)_{\delta}=\frac{1}{q_{2}}\left[\frac{\alpha_{2} \beta\left(x_{1}\right)_{\delta}\left(y_{1}\right)_{\delta} w}{w\left(y_{1}\right)_{\delta}+\left(y_{2}\right)_{\delta}}-d_{2}\right] .
\end{aligned}
$$

Hence once the optimal equilibrium, $\left(\left(x_{1}\right)_{\delta},\left(x_{2}\right)_{\delta},\left(y_{1}\right)_{\delta},\left(y_{2}\right)_{\delta}\right)$ is determined, the optimal harvesting effort $\left(E_{1}\right)_{\delta}$ and $\left(E_{2}\right)_{\delta}$ can be determined.

From (6.3), (6.4), (6.10), (6.11), (6.12) and (6.13), we found that $\lambda_{i}(t)$ where $i=1,2,3,4$, do not vary with time in optimal equilibrium. Hence they remain bounded as $t \rightarrow \infty$.

\section{Computer Simulation Discussion}

We have considered a system of two populations at different stage structure. The stability of the interior equilibrium point is studied and it is shown that the system is stable. We have shown the stability results analytically and also numerically. We can also considered a delayed model system to take into account of the gestational delay of the matured predator population. It is natural that the consumption on the prey by the predator needs some time to contribute to the biomass of the predator. So we use delay differential Equation (7.1) to study such phenomena.

$$
\frac{\mathrm{d} x_{1}}{\mathrm{~d} t}=r_{1} x_{1}-\frac{r_{1} x_{1}^{2}}{k_{1}}-\beta x_{1} y_{2}-\sigma_{1} x_{1}+\sigma_{2} x_{2}-q_{1} E_{1} x_{1}
$$




$$
\begin{gathered}
\frac{\mathrm{d} x_{2}}{\mathrm{~d} t}=r_{2} x_{2}-\frac{r_{2} x_{2}^{2}}{k_{2}}+\sigma_{1} x_{1}-\sigma_{2} x_{2} \\
\frac{\mathrm{d} y_{1}}{\mathrm{~d} t}=\frac{\alpha_{1} \beta x_{1} y_{2}^{2}}{w y_{1}+y_{2}}-\frac{\alpha_{2} \beta x_{1} y_{1} y_{2} w}{w y_{1}+y_{2}} \\
\frac{\mathrm{d} y_{2}}{\mathrm{~d} t}=\frac{\alpha_{2} \beta x_{1}(t-\tau) y_{1} y_{2} w}{w y_{1}+y_{2}}-d_{2} y_{2}-q_{2} E_{2} y_{2} .
\end{gathered}
$$

Delay differential equation models are studied extensively in the study of several ecological systems by $\mathrm{K}$. Das and N. H. Gazi [26,27]. All those systems are two and three dimensional systems. Since the present model is four dimensional system, the analytical study of the system is difficult to tractable and the expression for the delay parameter values will be complicated for which the system is stable. So, we solve the system numerically only. The numerical solutions are shown in the Figures 2-5. The Figures 2 and 3 show the stable solution of

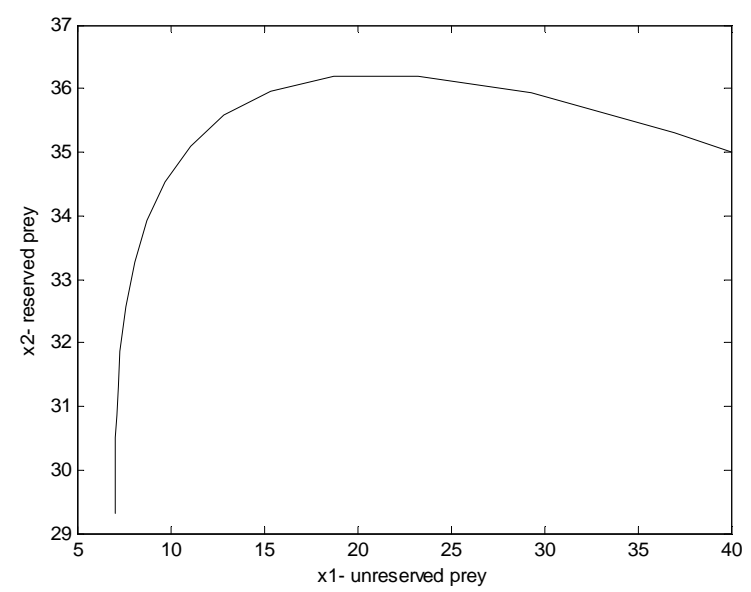

Figure 2. The stable phase portrait of prey in unreserved zone verses prey in reserved zone for delayed model system (7.1).
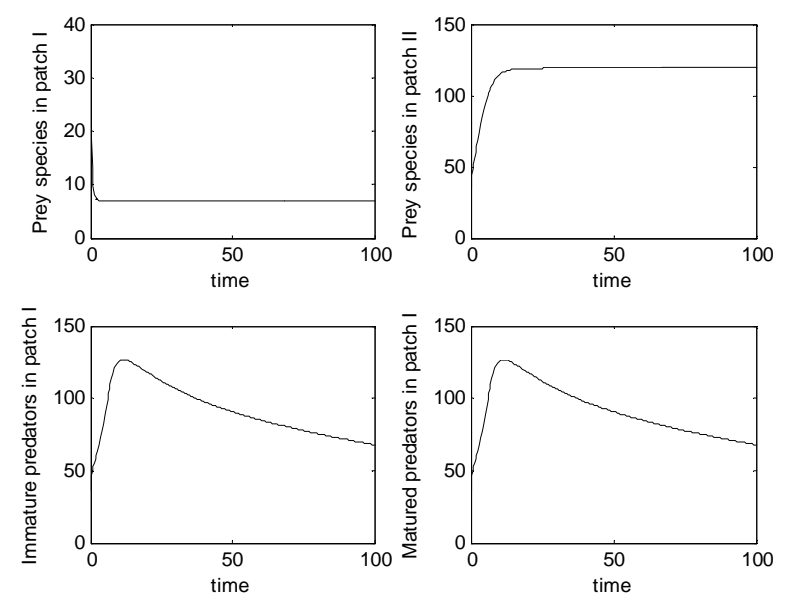

Figure 3. The stable time series evolution of the prey and predator populations of the delay model system (7.1) for $\tau<$ 10.
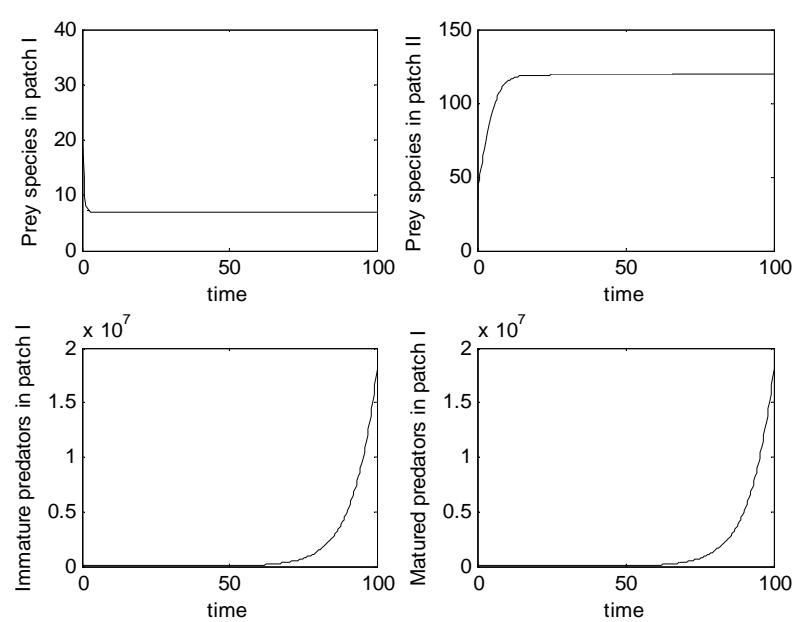

Figure 4. The unstable time series evolution of the prey and predator populations of the delay model system (7.1).

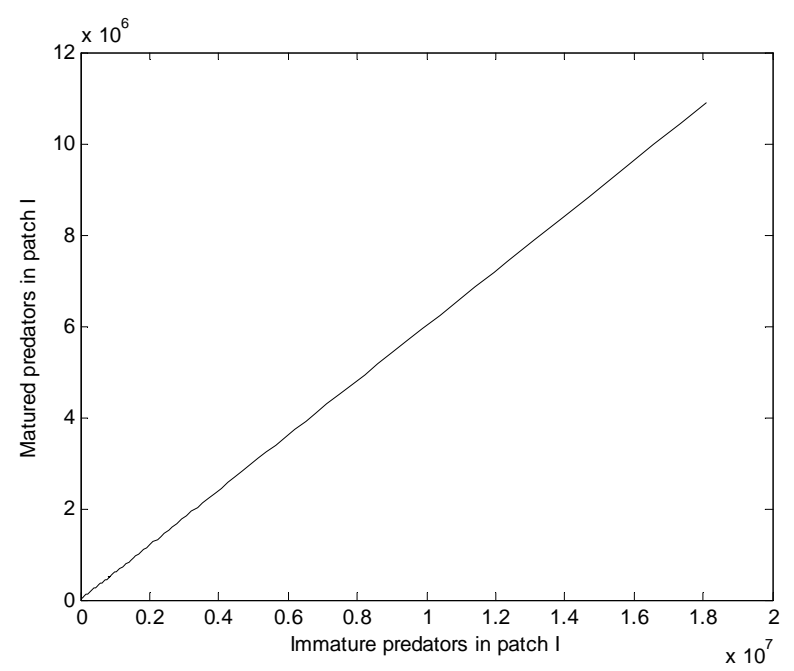

Figure 5. Unstable phase-portrait of the predator populations for the delayed model system (7.1) with delay parameter value $\tau \geq 10$.

the populations for $\tau \leq 10$ while the Figures 4 and 5 show that the system is unstable for $\tau>10$.

\section{Acknowledgements}

Authors are thankful to the reviewers for their valuable constructive comments and suggestions to improve this paper.

\section{References}

[1] F. Brauer and A. C. Soudack, "Stability Regions and Transition Phenomena for Harvested Predator-Prey Systems," Journal of Mathematical Biology, Vol. 7, No. 4, 1979, pp. 319-337. doi:10.1007/BF00275152

[2] F. Brauer and A. C. Soudack, "Stability Regions in Pre- 
dator-Prey Systems with Constant-Rate Prey Harvesting," Journal of Mathematical Biology, Vol. 8, No. 1, 1979, pp. 55-71. doi:10.1007/BF00280586

[3] G. Dai and M. Tang, "Coexistence Region and Global Dynamics of a Harvested Predator-Prey System," SIAM: SIAM Journal on Applied Mathematics, Vol. 58, No. 1, 1998, pp.193-210. doi:10.1137/S0036139994275799

[4] M. R. Myerscough, B. E. Gray, W. L. Hograth and J. Norbury, "An Analysis of an Ordinary Differential Equation Model for a Two-Species Predator-Prey System with Harvesting and Stocking," Journal of Mathematical Biology, Vol. 30, 1992, pp. 389-401.

[5] K. S. Chaudhuri and S. S. Ray, "On the Combined Harvesting of a Prey-Predator System," Journal of Biological Systems, Vol. 4, No. 3, 1996, pp. 373-389. doi:10.1142/S0218339096000259

[6] A. W. Leung, "Optimal Harvesting Co-Efficient Control of Steady State Prey-Predator Diffusive Volterra-Lotka Systems," Applied Mathematics \& Optimization, Vol. 31, No. 2, 1995, pp 219-241. doi:10.1007/BF01182789

[7] C. W. Clark, "Mathemalical Bioeconomics: The Optimal Management of Renewable Resources," John Wiley and Sons, New York, 1979.

[8] S. A. Levin, T. G. Hallam and J. L. Gross, "Applied Mathematical Ecology," Springer-Verlag, Berlin, 1989.

[9] W. G. Aiello and H. I. Freedman, "A Time Delay Model of Single Species Growth with Stage Structure," Mathematical Biosciences, Vol. 101, No. 2, 1990, pp. 139-153. doi:10.1016/0025-5564(90)90019-U

[10] H. I. Freedman and K. Gopalsammy, "Global Stability in Time-Delayed Single Species Dynamics," Bulletin of Mathematical Biology, Vol. 48, No. 5-6, 1986, pp. 485492.

[11] G. Rosen, "Time Delays Produced by Essential Nonlinearity in Population Growth Models," Bulletin of Mathematical Biology, Vol. 49, No. 2, 1987, pp. 253-255.

[12] M. E. Fisher and B. S. Goh, "Stability Results for Delayed Recruitment Models in Population Dynamics," Journal of Mathematical Biology, Vol. 19, No. 1, 1984, pp. 147-156. doi:10.1007/BF00275937

[13] M. Mesterton-Gibbons, "On the Optimal Policy for the Combined Harvesting of Predator and Prey," Natural Resource Modeling, Vol. 3, 1988, pp. 63-90.

[14] C. W. Clark, "Mathematical Bioeconomics: The Optimal Management of Renewable Resources," Wiley, New York, 1976.

[15] K. S. Chaudhuri, "A Bio Economic Model of Harvesting of a Multi Species Fishery," Ecological Modelling, Vol. 32, No. 4, 1986, pp. 267-279.

\section{doi:10.1016/0304-3800(86)90091-8}

[16] D. L. Ragozin and G. Brown, "Harvest Policies and Non Market Valuation in a Predator Prey System," Journal of Environmental Economics and Management, Vol. 12, No. 2, 1985, pp. 155-168. doi:10.1016/0095-0696(85)90025-7

[17] A. Hastings, "Global Stability of Two Species Systems," Journal of Mathematical Biology, Vol. 5, 1978, pp.399403.

[18] X.-Z. He, "Stability and Delays in a Predator-Prey System," Journal of Mathematical Analysis and Applications, Vol. 198, No. 2, 1996, pp. 355-370. doi:10.1006/jmaa.1996.0087

[19] B. S. Goh, "Global Stability in Two Species Interactions," Journal of Mathematical Biology, Vol. 3, No. 3-4, 1976, pp. 313-318. doi:10.1007/BF00275063

[20] W. G. Aiello and H. I. Freedman, "A Time Delay Model of Single Species Growth with Stage Structure," Mathematical Biosciences, Vol. 101, No. 2, pp. 139-153. doi:10.1016/0025-5564(90)90019-U

[21] W. G. Aiello, H. I. Freedman and J. Wu, "Analysis of a Model Representing Stage Structured Population Growth with State-Dependent Time Delay," SIAM: SIAM Journal on Applied Mathematics, Vol. 52, No. 3, 1992, pp. 855869.

[22] T. K. Kar and M. Swarnakamal, "Influence of Prey Reserve in a Prey-Predator Fishery," Non-Linear Analysis, Vol. 65, No. 9, 2006, pp.1725-1735.

[23] W. Wang and L. Chen, "Optimal Harvesting Policy for Single Population with Periodic Coefficients," Mathematical Biosciences, Vol. 152, No. 2, 1998, pp. 165-177. doi:10.1016/S0025-5564(98)10024-X

[24] R. Zhang, J. F. Sun and H. X. Yang, "Analysis of a PreyPredator Fishery Model with Prey Reserve," Applied Mathematical Sciences, Vol. 50, No. 1, 2007, pp. 24812492.

[25] W. D. Wang, Y. Takeeuchi,Y. Saito and S. Nakaoka, "Prey-Predator System with Parental Care for Predators," Journal of Theoritical Biology, Vol. 241, No. 3, 2005, pp. 451-458. doi:10.1016/j.jtbi.2005.12.008

[26] K. Das, N. H. Gazi, "Structural Stability Analysis of an Algal Bloom Mathematical Model in Trophic Interaction," International Journal of Non-linear Ananlysis: Real World Applications, Vol. 11, No. 4, 2010, pp. 21912206.

[27] N. H. Gazi and K. Das, "Control of Parameters of a Delayed-Diffusive Autotroph-Herbivore System," International Journal of Biological System, Vol. 18, No. 2, 2010, pp. 509-529. 